\title{
FAMILIA COMO UNIDAD CORONARIA PRIMARIA DE LAS PERSONAS HIPERTENSAS O DIABÉTICAS TIPO II
}

\author{
FAMILY AS A PRIMARY CORONARY UNIT OF HYPERTEN- \\ SIVE OR DIABETIC TYPE II PEOPLE
}

\author{
Diana Marcela Rengifo Arias ${ }^{1 *}$, Fabián Alexis Ropero Giraldo ${ }^{1}$ \\ 1. Programa Enfermería - Facultad Ciencias de la Salud - Universidad del Quindío. \\ *Correspondencia del Autor: Diana M. Rengifo. E mail: dmrengifoa@uniquindio.edu.co
}

\section{RESUMEN}

Los pacientes crónicos, presentan mayor riesgo de desarrollar eventos cardiovasculares que comprometan fatalmente su integridad física, poniendo en riesgo su vida, la mayoría de estos casos ocurren en el domicilio. Objetivo. Determinar la capacidad de respuesta de los familiares, de las personas que viven con hipertensión arterial o diabetes mellitus tipo 2 ante eventos cardiovasculares que comprometen potencialmente la vida de su familiar. Metodología. Estudio cuantitativo, descriptivo de corte trasversal empleando un muestreo probabilístico, aleatorio simple; la población fueron familias de personas que asisten al programa y control de riesgo cardiovascular. Resultados. La mayoría de los participantes fueron mujeres $(67,5 \%)$, estudiantes $(48,2 \%)$, de parentesco de primer grado de consanguinidad (hijos $48,7 \%)$, en una edad de 21 a 30 años $(34,3 \%)$ y con nivel educativo profesional $(48,0 \%)$; el $55 \%$ de las personas interrogadas refirió no conocer los signos asociados a estos eventos, solo el 40,2\% de ellos manifestaron sentirse en capacidad de identificar el evento, sin embargo, ninguno de ellos identifican la "no respuesta al llamado" como una condición asociada, solo el 0,5\% reconoció la secuencia correcta para actuar como primeros respondientes (activación del sistema de emergencia- Compresiones cardíacas- Desfibrilación precoz). El 85,5\% refirió no conocer un desfibrilador externo automático. Conclusión: no se encuentran en la capacidad de proveer maniobras de RCP en el caso de que sus familiares lo requirieran, puesto que existen grandes vacíos en el conocimiento sobre las actividades que deben desarrollar, además manifiestan no sentirse competentes para actuar en consecuencia con la situación.

Palabras claves: Familia, Resucitación Cardiopulmonar, Cuidado de Enfermería, Enfermedades Cardiovasculares, Hipertensión, Diabetes Mellitus Tipo 2, Infarto. (Fuente: DeCS, BIREME).

Cómo citar:

Rengifo, D.M, Ropero, F.A. (2017) Familia como unidad conoraria primaria de las personas hipertensas o diabéticas tipo II. Revista de Investigaciones de la Universidad del Quindío. Vol 29, pp.7-15. 


\begin{abstract}
Chronic patients have a higher risk of suffering cardiovascular events that fatally compromise their physical integrity, placing their lives at risk. Most of these cases occur at home. Objective. This work sought to determine the response capacity of relatives of individuals with high blood pressure or diabetes mellitus type 2 on the event of cardiovascular episodes that potentially compromise the lives of their family members. Methodology. This was a quantitative, descriptive, cross-sectional study using probabilistic, simple random sampling. The population selected were relatives of individuals attending the program and control of cardiovascular risk. Results. Most of the participants were women (67.5\%), students (48.2\%), kinship in the first degree of consanguinity (offspring 48.7\%), in an age range from 21 to 30 years (34.3\%), and professional educational level (48\%); 55\% of those interviewed reported not knowing the signals associated to these events; only $40.2 \%$ of them stated feeling able to identify the event; however, none of them identifies the "non-response to the call" as an associated condition; only $0.5 \%$ recognized the correct sequence to act as first respondents (activation of the emergency system - cardiac compressions - early defibrillation). Of the participants, $85.5 \%$ stated not knowing an automatic external defibrillator. Conclusion. The participants are not in the capacity to engage in CPR maneuvers in case their relatives require it, given the existence of large voids in knowledge of the activities they have to carry out. Additionally, they state they do not feel competent to act consequently with the situation.
\end{abstract}

Keywords: Family, Cardiopulmonary Resuscitation, Nursing Care, Cardiovascular Disease, Hypertension, Diabetes Mellitus Type 2, Stroke (Source: DeCS, BIREME).

\section{INTRODUCCIÓN}

La ingesta de alimentos poco saludables, el consumo de tabaco - alcohol, el estrés y el sedentarismo, son factores a los que la población se expone con mayor frecuencia, generando en el individuo una predisposición para el desarrollo de enfermedades crónicas no transmisibles (ECNT), las cuales se caracterizan por ser de lenta evolución y larga duración, dentro de ellas las enfermedades cardiovasculares (enfermedad coronaria y enfermedad cerebrovascular, enfermedad arterioesclerótica y enfermedad renal), el cáncer, las enfermedades respiratorias crónicas y la diabetes 1 reportan, según datos de la Organización Mundial de la Salud (OMS), más de 36 millones de muertes al año a nivel mundial. Para Colombia2, la diabetes mellitus y la enfermedad cardiovascular se encuentran dentro de las primeras causas de mortalidad asociada a ECNT en la población mayor de 45 años.

Los pacientes crónicos, tales como las personas que conviven con hipertensión arterial (HTA) o diabetes mellitus tipo 2 (DM2) presentan mayor riesgo de desarrollar eventos cardiovasculares que comprometan fatalmente su integridad física, poniendo en riesgo su vida. Por lo anterior, es importante determinar la capacidad de respuesta como unidad coronaria primaria de sus familiares, teniendo en cuenta que son ellos los que deberán actuar como primeros respondientes ante un evento cardiovascular fatal, brindando soporte vital básico (SVB) y desfibrilación precoz, garantizando una mejor sobrevida de su familiar.
El riesgo de padecer un evento cardiovascular potencialmente fatal incrementa en las personas que desarrollan ECNT y la probabilidad de sobrevivir a uno de estos sigue siendo baja, dependiendo de tres variables principalmente: el lugar donde se produce el evento, el tiempo que transcurre hasta que comienza la reanimación cardiopulmonar y el tipo de evento3. El inicio tardío o el no inicio de maniobras de SVB antes de la llegada de la ambulancia4 generan un aumento de la lesión isquémica y disminuye la posibilidad de lograr con éxito el retorno a la circulación espontanea, afectando principalmente órganos como el corazón y el cerebro. Al ocurrir la mayoría de estos eventos en entornos extra hospitalarios4-5, y siendo el hogar el lugar de mayor incidencia4, los familiares y testigos legos constituyen el primer nivel de atención para proveer SVB, sobre todo en aquellas familias donde uno de sus miembros convive con ECNT, lo cual significa que la familia debe estar entrenada para actuar como primeros respondientes, operacionalizando la cadena de supervivencia propuesta por la American Heart Association (AHA); si no se logra esta condición, el tiempo que transcurre hasta que comienza la reanimación cardiopulmonar aumenta, incidiendo directamente en la mortalidad asociada al evento o en el aumento de las secuelas asociadas al mismo.

La OMS6-1 estima que las ECNT constituyen la principal causa de mortalidad mundial y asegura que el $80 \%$ de éstas ocurren en países de ingresos medios y bajos, generando un gran impacto social, económico 
y humano, puesto que más de una cuarta parte de estas muertes afectan a las personas menores de 60 años, perdiendo, así, un gran recurso productivo en las microeconomías familiares y la macroeconomía social. Por cada minuto que una persona se encuentra experimentando un paro, sin recibir RCP de calidad, su pronóstico de sobrevida disminuye en un $10 \%$, con lo cual se deduce que pasados 10 minutos de soportar un paro cardio-respiratorio la posibilidad de retornar a la circulación espontanea es cero y al ser éste, el paro cardio-respiratorio, un evento que en la mayoría de los casos ocurre en el hogar, la familia -como testigos presenciales del hecho- se constituye como los primeros potenciales respondientes y como tales deben ser tenidos en cuenta, por ser un recurso valioso a la hora de servir como unidad coronaria primaria. La familia, al ser considerada como la unidad básica social, es la responsable de la estructuración de prácticas que propenden y mantienen la vida, se constituye como un grupo social primario, formado por individuos unidos por lazos sanguíneos, o de afinidad o de matrimonio, que interactúan y conviven en forma más o menos permanente y que, en general, comparten factores biológicos, sociales y sicológicos que pueden afectar a sus integrantes de forma individual o colectiva7. Es en la familia donde se genera y se resuelven la mayoría de los asuntos relacionados con el proceso de salud y enfermedad; es la principal fuente de creencias y hábitos de vida, son quienes reconocen las características comunes de las personas que en ella conviven, son los primeros que pueden determinar la presencia de signos y síntomas de alarma. Pueden iniciar actividades promotoras de salud, contribuir con el desarrollo, la adherencia al tratamiento y activar la atención temprana ante situaciones que ponen en riesgo la vida de sus miembros, es decir, constituyen un sistema esencial para garantizar prácticas de cuidado y autocuidado en el entorno primario de la persona que convive con ECNT y que debe de ser así valoradas por el personal asistencial a la hora de desarrollar acciones de promoción de la salud y prevención de la enfermedad en los diferentes niveles de atención.

Esta situación no presenta un panorama consolador, puesto que el conocimiento claro sobre los factores de riesgo asociados a la patogenia de ambas enfermedades y el entendimiento de cómo estos pueden ser impactados mediante el desarrollo de estrategias educativas y de salud orientadas a la población general desde acciones contempladas dentro de las estrategia de prevención secundaria y terciaria en las cuales el conocimiento pleno de su enfermedad, la compren- sión de la importancia que tiene la adherencia al tratamiento farmacológico, la modificación de patrones de vida poco saludables, el reconocimiento de signos de alarma, el manejo temprano de los mismos y de las situaciones que pueden comprometer fatalmente la vida, constituyen un pilar importante para la disminución de la mortalidad asociada a estas patologías y al mejoramiento de la calidad de vida de las personas que viven con HTA o DM2. Acciones dentro de las cuales la familia y la comunidad toman un papel importante, al ser ellos testigos potenciales de eventos que pueden comprometer fatalmente la vida de las personas que viven con ECNT (específicamente HTA o DM2), por tener este grupo de personas un riesgo superior de experimentar eventos cardiovasculares letales, que aportan 17,3 millones de defunciones al año en la población de personas que viven con una ECNT6.

El presente estudio tuvo como objetivo determinar la capacidad de respuesta, como unidad coronaria primaria, de los familiares de las personas que viven con HTA o DM2 ante eventos cardiovasculares que comprometen potencialmente la vida de su familiar; para ello, se identificó el conocimiento de las personas sobre los signos de alarma de un evento cardiovascular fatal y la respuesta en SVB, se clasificaron las conductas reconocidas por los familiares y se evaluó la disponibilidad de un desfibrilador externo automático (DEA) en su entorno.

\section{MATERIALES Y MÉTODOS}

El presente estudio fue cuantitativo, exploratorio, de corte trasversal; permitió reconocer los conocimientos sobre signos de alarma de evento coronario, SVB y desfibrilación con DEA de las familias como unidad coronaria básica.

La población fueron las familias de las personas asistentes al programa y control de riesgo cardiovascular, diagnosticadas con hipertensión arterial sistémica y diabetes mellitus de una institución prestadora de servicios de salud en el primer nivel de atención de la ciudad de Armenia - Quindío, Colombia. La muestra fue probabilística aleatoria simple, considerando un nivel de confianza del 95\%, un error máximo de estimación del $5 \%$ y un efecto amortiguador de $10 \%$ sobre una población de referencia de 10.000 personas, para un total de 400 personas. 
Esta investigación se llevó a cabo bajo las directrices de la resolución 8430 de 1993; se obtuvo la aprobación de los comités de investigación y ética de la Universidad del Quindío, como se registra en el acta 024 2014. Se tomaron como criterios de inclusión: ser familiar de un paciente con diagnóstico de HTA o DM2, vinculación del familiar al programa de riesgo cardiovascular y/o a los controles de manejo de estas patologías y ser mayor de 18 años. Los criterios de exclusión fueron haber participado en alguna intervención educativa relacionada desarrollada por la Universidad del Quindío.

La información se recolectó por medio de un instrumento elaborado por los investigadores, validándolo mediante una prueba piloto realizada a una población similar, en busca de desarrollar dominio en la aplicación del instrumento, además de valorar la comprensión y claridad de las preguntas del mismo. Posterior a la obtención del registro de las personas vinculadas al programa de riesgo cardiovascular, se procedió a realizar una aleatorización simple para identificar los casos índices, quienes fueron localizados en su lugar de consulta, contactados y programados para visita en su domicilio, lugar donde se procedió a la obtención de la autorización de su familiar, por medio del consentimiento informado. La información fue analizada en una base de datos en Microsoft Excel 2011, previo vaciamiento y depuración de la misma; los resulta- dos obtenidos se describieron mediante medidas de tendencia central y distribución de frecuencias, relacionando aspectos como: datos demográficos de la población, conocimientos sobre signos de alarma, conocimientos sobre SVB, uso del DEA y disponibilidad del mismo.

\section{RESULTADOS}

Para el análisis de la información, se empleó la estadística descriptiva univariada, encontrando que la mayoría de los participantes fueron mujeres $(67,5 \%)$, estudiantes $(48,2 \%)$, de parentesco de primer grado de consanguinidad (hijos 48,7\%), con una edad de 21 a 30 años $(34,3 \%)$ y un nivel educativo profesional $(48,0 \%)$.

Respecto a los síntomas identificados como signos de alarma para los eventos cardiovasculares, el 55\% de las personas interrogadas refirió no conocer los signos asociados a estos eventos, mientras que el $45 \%$ restante manifestó como síntoma prioritario la cefalea intensa $(20.5 \%)$, dolor u opresión en el pecho (20\%), desorientación-agitación $(20 \%)$, la somnolencia o profundo adormecimiento $(9,4 \%)$. Cabe resaltar que cambios como el habla incongruente e incomprensible, dificultad para respirar y mareos no fueron reconocidos por los participantes como signo de alarma (Gráfica 1).

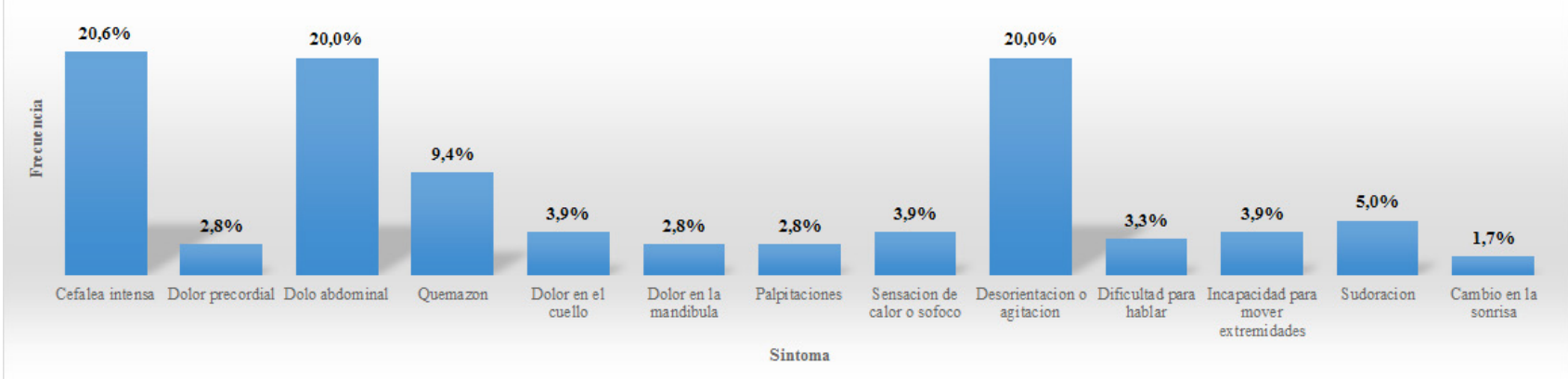

Gráfica 1. Distribución de la población, según síntomas identificado como signo de alarma. Fuente: elaboración propia

Con relación a la capacidad de reconocer si una persona se encuentra cursando por un paro cardiorrespiratorio, sólo el 40,2\% de ellos manifestó sentirse en capacidad de identificar el evento; de estas 161 personas, sólo el 28,5\% identifica una de las condiciones asociadas al paro cardiorrespiratorio (respiraciones anormales); sin embargo, ninguno de ellos identifi- ca la "no respuesta al llamado" como una condición asociada al paro cardiorrespiratorio. El 65\% de ellos identifica que la persona en paro cardíaco "ni se mueve, ni respira", el 1,8\% refirió como condición asociada el "no respira"; por su parte, el "no tener pulso" fue identificada como condición en el 1,2\% de los casos, igual porcentaje no respondió (Gráfica 2.). 


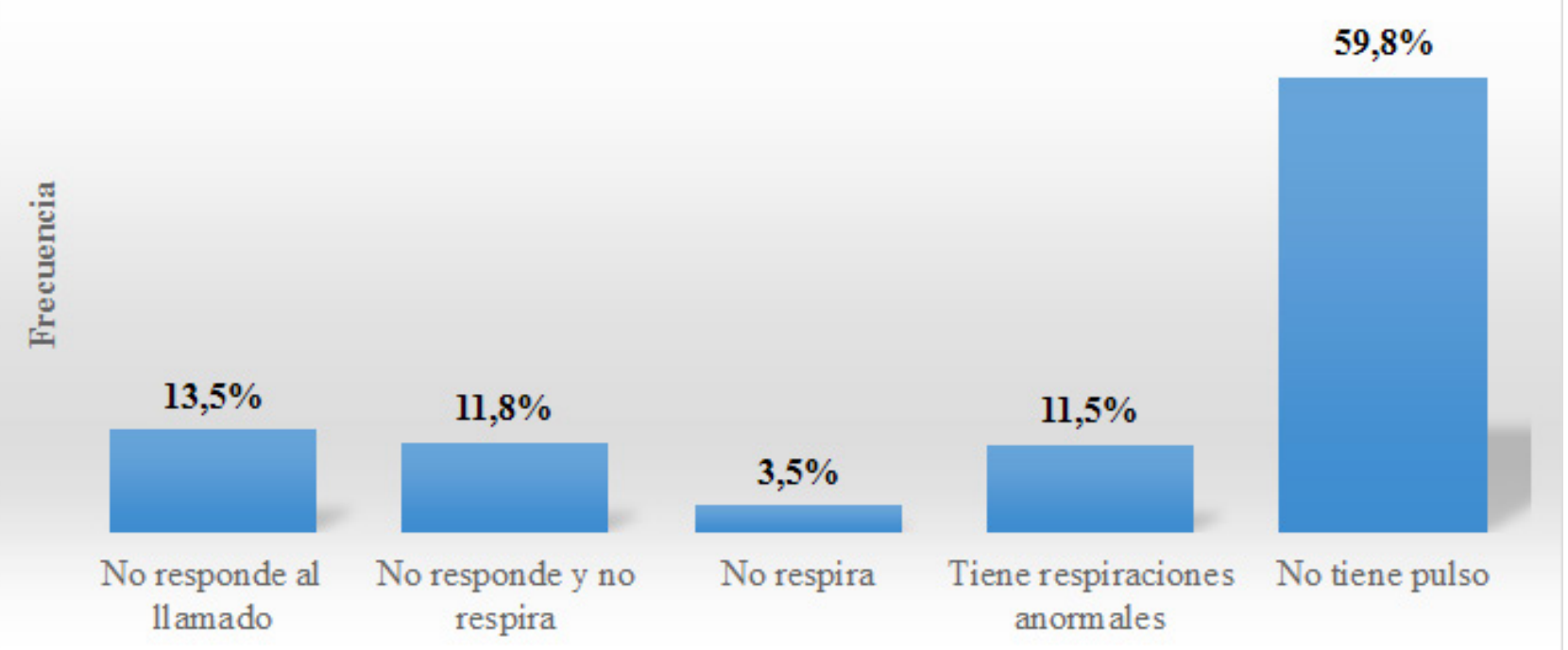

Gráfica 2. Distribución de la población, según síntomas asociados al paro cardiorrespiratorio. Fuente: elaboración propia

Dentro de las acciones identificadas como primordiales para brindar SVB, la mayoría de las personas identificó sólo una acción de la cadena de supervivencia y sólo el $0,5 \%$ reconoció la secuencia correcta para actuar como primeros respondientes (activación del sistema de emergencia- compresiones cardíacasdesfibrilación precoz). De la mayoría mencionada, el
$64 \%$ identificó como actuación prioritaria la activación del sistema de emergencia médico, seguido por el 27,7\% que manifestó que la acción prioritaria era realizar el traslado inmediato a un centro hospitalario; $5,75 \%$ no respondió a la pregunta realizada y el $2 \%$ restante refirió que lo más importante era iniciar compresiones cardíacas (Gráfica 3).

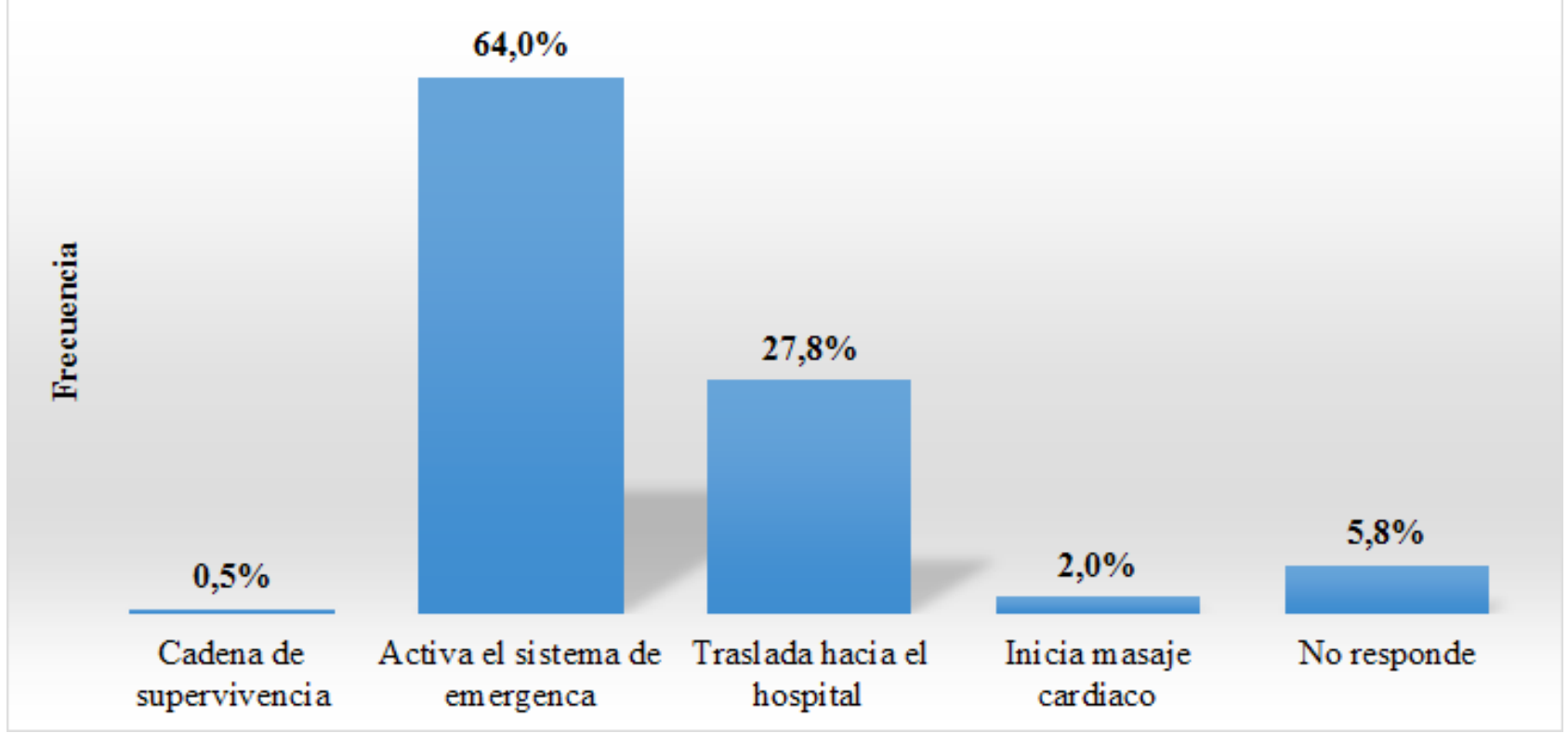

Gráfica 3. Distribución de la población, según actuación ante un paro. Fuente: elaboración propia

Ante la pregunta del conocimiento de un desfibrilador externo automático, el $85,5 \%$ de los familiares refirió no conocer el dispositivo; $13,75 \%$ manifestó conocerlo, mientras el 0,75\% no respondió. El 10,2\% de los familiares manifestó conocer el sitio donde se encuentra ubicado el DEA más cercano a su hogar, el $89 \%$ manifestó no conocer su ubicación y el $0,75 \%$ restante no respondió. De las personas que manifestaron conocer la ubicación del DEA más cercano a su hogar, el 78\% registró como ubicación el hospital, $14,6 \%$ no respondió, mientras que el $4,8 \%$ y el $2,4 \%$ manifestó que los lugares donde se hallaban los dispositivos son el centro comercial y un centro de educación superior, respectivamente. 
El 83\% de los familiares manifestó que no se consideran competentes para brindar la ayuda que su familiar requiere en maniobras de SVB. Sin embargo, del total de los familiares que manifestaron sentirse capacitados, 39,6\% expresó no tener clara la secuencia de acciones que ejecutarían, el 32,3\% refirió que podrían suministrar ventilación boca a boca, mientras que el $26,4 \%$ se consideró en capacidad de realizar compresiones cardíacas; el 1,4\% restante se consideró en capacidad de solicitar ayuda, brindar respiración y verificar pulso.

Para corroborar la capacidad manifestada por los participantes, para apoyar en SVB a sus familiares, se les solicitó identificar las acciones que correspondían a la cadena de supervivencia de una serie de imágenes, de las cuales sólo el 1,4\% pudo identificar la secuencia correcta de actividades, el 95,5\% identificó secuencias incorrectas, mientras que el $2,9 \%$ no completó el cuestionario. Posterior a esto, las actividades contempladas dentro de la cadena de supervivencia fueron fragmentadas por eslabones.

En cuanto al reconocimiento inmediato del paro, el $42,6 \%$ de los familiares respondió que la acción a realizarse era verificar si la persona responde llamándolo, golpeándolo suavemente y comprobando si hay movimiento torácico o respiración normal, tardando máximo 10 segundos. Cuando se interrogó sobre la forma en la cual realizarían las compresiones torácicas, el 38,2\% refirió que colocarían sus manos en el centro del tórax, compresiones rápidas, fuertes al menos 100 minutos; este mismo porcentaje consideró que se puede realizar sólo compresiones torácicas. El $26,4 \%$ de los informantes reportó que siempre se tiene que suministrar dos respiraciones, aun si no se cuenta con los elementos de protección personal.

En relación a la posibilidad de acceder a un DEA y sobre la forma de utilizarlo, la mayoría $(32,3 \%)$ de los participantes manifestó no tener claro los pasos que se deberían realizar para utilizar el dispositivo; el 29,4\% consideró que sólo puede ser manipulado por personal con entrenamiento en salud que trabaje en centros hospitalarios, mientras que el $17,64 \%$ seleccionó la secuencia correcta para su utilización. E1 $13,2 \%$ de los participantes no respondió a la pregunta y el 7,35\% restante informó que primero fijarían los electrodos al pecho del paciente y posteriormente encendería el dispositivo. Por último, el $85,2 \%$ de los familiares manifestó no considerarse en capacidad de manipular un DEA, el 3,4\% se abstuvo de contestar a la pregunta, mientras que el grupo restante $(13,7 \%)$ consideró poder manipularlo.

\section{DISCUSIÓN}

El propósito de la presente investigación fue determinar la capacidad de respuesta de los familiares de las personas que conviven con HTA y DM2 ante eventos que comprometen potencialmente la vida de su familiar. Dado que el diseño del presente estudio es exploratorio y ante los escasos estudios reportados en relación con este tema, la discusión de los datos se centrará en la relación y contrastación de la respuesta proporcionada por los participantes. Así, a pesar de que el $55 \%$ de los participantes manifestó no estar en capacidad de reconocer los signos de alarma de eventos cardiovasculares, el 58.1\% de ellos logró identificar la mayoría de los síntomas relacionados con bajo gasto cardíaco, enfermedad cerebrovascular e infarto agudo de miocardio, lo cual permitiría que las familias promovieran una consulta oportuna a los servicios de urgencias en el caso de presenciar la aparición de los síntomas; sin embargo, la baja percepción sobre su capacidad de reconocimiento es la razón por la cual se hace importante que dentro de las estrategias educativas desarrolladas con la población de alto riesgo cardiovascular, los enfermeros involucren a las familias y la red de apoyo de los pacientes, lo cual podría generar no sólo un mayor nivel de confianza sobre las capacidades para el reconocimiento de la situación del riesgo, sino que permitiría intervenir oportunamente para disminuir las posibilidades del desarrollo del paro.

En relación a la posibilidad de reconocer un paro cardiorrespiratorio, un menor número de familiares (40,2\%) expresó sentirse capacitado; pero, a pesar de esta percepción, los conocimientos para identificar la situación no son suficientes, puesto que la indicación propuesta por AHA8 señala que se considera que una persona se encuentra en paro cardiorrespiratorio cuando no responde al llamado - no respira con normalidad; ante lo cual, ninguno de los participantes identificó la combinación de estas condiciones y sólo el $28,5 \%$ de los familiares identificó "el no respirar" como una situación que le permitiría reconocer la parada cardíaca. Esta situación requiere intervención inmediata, puesto que por cada minuto que una persona se encuentra en paro cardiorrespiratorio, sin recibir SVB, se está comprometiendo su posibili- 
dad de sobrevida en un $10 \%$, por lo que el reconocimiento precoz del evento juega un papel primordial en la atención de estos pacientes. La AHA9 calcula que la posibilidad de sobrevida de una persona que recibe SVB oportunamente por un testigo presencial del paro, es tres veces mayor que aquellos que fueron asistidos sólo hasta que recibieron ayuda médica; de ahí, la necesidad imperante de capacitar a las comunidades y los núcleos cercanos de las personas que presentan alto riesgo cardiovascular, en el marco de las estrategias de prevención secundaria y dentro de las actividades que se realizan desde el plan de intervenciones colectivas, estrategias que han sido implementadas en otras poblaciones, como estudiantes daneses 10 y población en general de Portugall1 y que han demostrado generar un impacto significativo en la posibilidad de sobrevida.

Cabe resaltar que sólo el $0,5 \%$ de las participantes seleccionó la secuencia correcta para brindar SVB, no obstante, aunque la mayoría de los participantes (64\%) no reconoce la secuencia, sí mencionan como acción prioritaria la activación del sistema de emergencias medico (SEM), desde donde podrían recibir las indicaciones necesarias para brindar soporte a su familiar mientras llega el equipo de salud. Se requiere, entonces, de la organización eficiente de los sistemas de emergencia para cada una de las localidades y del traslado oportuno de equipos de atención prehospitalaria hacia el lugar del evento para, así, apoyar de forma eficiente y eficaz a las personas que cursan por un paro cardiorrespiratorio, con el fin de impactar en la sobrevida; en esta organización, el profesional de enfermería juega un papel fundamental puesto que sus habilidades administrativas, educativas, asistenciales e investigativas le permitirían desarrollar programas que impacten en la sobrevida de las poblaciones desde los diferentes niveles de atención de salud, promoviendo -por demás- espacios de crecimiento y desarrollo curricular en los cuales las instituciones académicas podrían responder no sólo a su deber como formadoras de profesionales, sino al impacto social que deben realizar en las comunidades.

Además, el 85,5\% de los participantes refirió no conocer un DEA, situación que no limitaría la realización de maniobras de RCP bajo la estrategia de sólo manos, hasta que llegue ayuda especializada, que tiene como factor determinante un arribo oportuno estimado en menos de diez minutos; sin embargo, en países con mayor aceptación del uso del DEA, las tasas de sobrevida son más altas luego de episodio de paro cardíaco12, lo cual incluye la disponibilidad y fácil acceso a los dispositivos por parte de la población en general que presencia un paro cardíaco en el lugar del evento.

El desconocimiento de habilidades de SVB no es exclusivo de la población de Armenia, puesto que en Huesca también se reportaron datos bajos de capacidad para el desarrollo de maniobras de RCP por parte de familiares13. Cabe resaltar que un alto porcentaje de ellos manifestó haber participado con antelación en capacitaciones para primeros respondientes. Aunque el porcentaje para esa población continua siendo bajo a lo esperado, existe diferencia con los resultados del presente estudio, que quizás puede ser asociada al desarrollo de las capacitaciones.

A pesar de no contar con instrumentos validados para determinar la capacidad de la familia para responder ante una situación que comprometa la vida de su familiar desarrollando habilidades de SVB, el presente estudio permite reconocer la realidad que experimentan las familias y las personas que conviven con HTA y DM2 ante el riesgo de experimentar o presenciar un paro cardiorrespiratorio. Este conocimiento podrá ser empleado como línea de base para la estructuración y el desarrollo de estrategias de prevención secundarias, dentro de las cuales los profesionales de enfermería podrán desempeñar infinidad de tareas que implican el desarrollo de actividades de gestión, administración, investigación, academia e intervención directa, no sólo en los diferentes niveles de atención de salud, sino dentro del sistema mismo, puesto que la operacionalización de la cadena de supervivencia y la estrategia de SVB implica la construcción de voluntad política, institucional y económica.

\section{CONCLUSIONES}

Los resultados reportados en esta investigación permiten determinar que las familias de las personas que conviven con HTA y DM2 de la ciudad de ArmeniaQuindío (Colombia) no se encuentran en la capacidad de proveer maniobras de RCP en el caso de que sus familiares lo requirieran, puesto que existen grandes vacíos en el conocimiento sobre las actividades que deben desarrollar, además manifiestan no sentirse competentes para actuar en consecuencia con la situación. 
Resulta importante el desarrollo e implementación de actividades educativas inmersas dentro de las estrategias de prevención secundaria con las cuales impactar de forma positiva en las tasas de morbimortalidad asociadas a paro cardiorrespiratorio, mejorando la posibilidad de sobrevida de aquellos pacientes que experimentan situaciones de paro en ambientas extra hospitalarios.

Los enfermeros, por su formación profesional, poseen altas competencias para el diseño, administración, desarrollo y gestión de programas de primeros respondientes, desde los cuales se podría construir, por parte del paciente y de sus familiares, conocimiento pleno sobre la enfermedad, adherencia al tratamiento farmacológico y no farmacológico, reconocimiento de signos de alarma, manejo temprano de los mismos y de las situaciones que pueden comprometer fatalmente la vida de las personas que conviven con HTA y DM2.

\section{RECOMENDACIONES}

Es importante desarrollar estudios experimentales que puedan demostrar el impacto que tienen las intervenciones educativas sobre el desarrollo de habilidades, como primeros respondientes en la población sin entrenamiento en salud, además de generar estudios que permitan contrastar los índices de casos de personas que experimentaron paros cardiorrespiratorios y que recibieron soporte vital in situ en el contexto regional y la sobrevida de ellos respecto a quienes no fueron reanimados por testigos presenciales.

\section{AGRADECIMIENTOS}

Los autores agradecen a la Universidad del Quindío por proporcionar el recurso para el desarrollo de esta investigación y a cada una de las familias que atendieron el llamado durante el desarrollo de la misma.

\section{BIBLIOGRAFÍA}

1. Organización Mundial de la Salud. Enfermedades cardiovasculares [en línea]. Washington: OMS, 2013. [Consultado Ene. 04, 2013]. Disponible en internet: http://www.who.int/mediacentre/factsheets/ fs $317 /$ es/.

2. COLOMBIA. Situación de salud en Colombia: Indicadores básicos 2011 [en línea]. Bogotá: El Ministerio. 2011. Disponible en Internet: http://www.minproteccionsocial.gov.co/salud/Paginas/INDICADORESBASICOSSP.aspx.

3. KAPLAN, Roberta; SCHUB, Tanja. Paro cardíaco (muerte cardíaca súbita): evaluación de los riesgos y diagnóstico [en línea]. En: CINAHL Nursing Guide. 2012, Ago, vol. 31, p. 2. [consultado Ene. 09, 2014]. Disponible en Internet:http://search.ebscohost.com/login.aspx?direct=true\&db=nre\&AN=SPA $5000004563 \&$ lang $=$ es\&site $=$ nrc-spa.

4. BALLESTEROS-PEÑA, Sendoa et al. Factores asociados a la mortalidad extra hospitalaria de las paradas cardiorrespiratorias atendidas por unidades de soporte vital básico en el País Vasco [en línea]. En: Revista Española de Cardiología. 2013, vol. 66 no.4, p. 269-274. [consultado Dic. 22, 2013]. Disponible en Internet: http://http://www.revespcardiol.org.

5. AGUDELO, Alejandro. Actualización en reanimación: Parte I [en línea]. Departamento de anestesiología, Universidad del Valle. 2009, p. 1-5. [consultado Dic. 22, 2013]. Disponible en Internet: https:// scare-multimedia.s3.amazonaws.com/.../186_reanimacion_y_cuidados.

6. Organización Mundial de la Salud. Informe sobre la situación mundial de las enfermedades no transmisibles [en línea]. Ginebra: OMS, 2011. [Consultado Ene. 07, 2014]. Disponible en internet: http://www. who.int/nmh/publications/ncd_report_summary_es.pdf.

7. DIDONI, Marisa et al. La familia como unidad de atención [en línea]. Disponible in internet: http:// ecaths1.s3.amazonaws.com/cursopami/148478073.familia.pdf.

8. Asociación Americana del Corazón. Soporte vital cardiovascular básico: libro del proveedor. $1^{\mathrm{a}}$ ed. Estados Unidos: Integracolor Ltd. 2012.

9. Asociación Americana del Corazón. Paro cardíaco [en línea]. Washington: AHA, 2009. [consultado Ene. O4, 2013]. Disponible en: http://esamericanheart.convertlanguage.com/presenter. jhtml?identifier $=4481$.

10. Aaberg A, Larsen C, Rasmussen B, Hansen C, Larsen J. Basic life support knowledge, self-reported skills and fears in Danish high school students and effect of a single 45-min training session run by junior doctors; a prospective cohort study. Scandinavian Journal of Trauma, Resuscitation and Emer- 
gency Medicine [en línea]. 2014 Abr [consultado Ene. O4, 2013]. Disponible en: http://www.sjtrem. com/content/pdf/1757-7241-22-24.pdf.

11. Tabares A, Pedro M, Urbano J. Ausência de formação em suporte básico de vida pelo cidadão: um problema de saúde pública? Qual a idade certa para iniciar?. Rev Port Saúde pública [en línea]. 2016; 34(1):101-104 [consultado 2016 Ene 04]. Disponible en: http://www.sciencedirect.com/science/article/pii/S0870902515000450.

12. COGOLLO, David et al. Atención pre hospitalaria de paro cardiorrespiratorio con y sin desfibrilador externo automático -DEA. Informe final de investigación [en línea]. Medellín, Colombia: Universidad CES. 2010. Disponible en internet: http://bdigital.ces.edu.co:8080/dspace/bitstream/.../1/INVESTIGACION.pdf.

13. Arrazola Alberdi O, García Moyano LM, Sanz Pérez L. Los familiares que habitan con enfermos cardíacos de Huesca, ¿están preparados para hacer frente a una parada cardiorrespiratoria? Enfermería Global [en línea]. 2015; 14(4):286-295 [consultado 2016 Ene 04]. Disponible en: http://revistas.um.es/ eglobal/article/view/220091/181611. 